\title{
Soil plasticity with a different porosity
}

\author{
Sergii Klovanych ${ }^{1}$, Leszek Malyszko ${ }^{1, *}$ \\ ${ }^{1}$ Faculty of Geodesy, Geospatial and Civil Engineering \\ University of Warmia and Mazury in Olsztyn,ul. Prawocheńskiego 15, 10-720 Olsztyn, Poland
}

\begin{abstract}
The model of soils with different porosity in the framework of the associated theory of plasticity is presented The single analytical function describes the loading surface in the stress space. The deformational hardening/softening and the phenomenon of dilatancy during plastic flow are incorporated in the model. The triaxial compression tests are simulated and compared with the experimental results for different values of the void ratio and initial hydrostatic stresses.
\end{abstract}

\section{Introduction}

In accordance with the classical plastic flow theory total strain rate $\dot{\boldsymbol{\varepsilon}}$ is decomposed into an elastic part $\dot{\boldsymbol{\varepsilon}}^{e}$ and a plastic part $\dot{\boldsymbol{\varepsilon}}^{p l}$, i.e. $\dot{\boldsymbol{\varepsilon}}=\dot{\boldsymbol{\varepsilon}}^{e}+\dot{\boldsymbol{\varepsilon}}^{p l}$. The plastic strains $\dot{\boldsymbol{\varepsilon}}^{p l}$ are determined by the flow rule $\dot{\boldsymbol{\varepsilon}}^{p l}=\dot{\lambda} \mathbf{g}$, where $\mathbf{g}$ is the second-order tensor of the plastic flow direction that is normal to a plastic potential surface in a stress space and the plastic multiplier $\dot{\lambda}$ determines the magnitude of the plastic strains. Adopting other governing equations, the stress rate $\dot{\boldsymbol{\sigma}}$ can be finally written in terms of the total strain rate $\dot{\boldsymbol{\varepsilon}}$ as

$$
\dot{\boldsymbol{\sigma}}=\mathbf{D}^{e p} \cdot \dot{\boldsymbol{\varepsilon}} \equiv\left[\mathbf{D}-\frac{(\mathbf{D} \cdot \mathbf{f}) \otimes(\mathbf{D} \cdot \mathbf{g})}{\mathbf{f} \cdot \mathbf{D} \cdot \mathbf{g}+H}\right] \cdot \dot{\boldsymbol{\varepsilon}},
$$

where $\mathbf{D}$ is the elastic stiffness tensor. The double contractions of tensors are denoted by one dot, the symbol $\otimes$ denotes a tensor product and the tensor of tangent elastoplastic modulus or the elastoplastic continuum fourth-order operator $\mathbf{D}^{e p}$ is generally no symmetric for arbitrary $\mathbf{g}$, except the case of an associative flow rule $\mathbf{f}=\partial f / \partial \boldsymbol{\sigma}=\mathbf{g}$ which is here discussed.

Depending on the hardening/softening modulus $H$ and the loading function $f$ or the gradient tensor f, the different material response can be obtained in the constitutive equation (1), see [1].

For isotropic materials a surface of the loading function $f$ is usually given in the stress space and can be convenient expressed in terms of scalar invariants of the stress tensor $\boldsymbol{\sigma}$. Using the deviatoric stress tensor $\mathbf{s}$ and the standard decomposition of the stress tensor into

\footnotetext{
*Corresponding author: leszek.malyszko@uwm.edu.pl
} 
its spherical and deviatoric parts $\boldsymbol{\sigma}=\frac{1}{3}(\operatorname{tr} \boldsymbol{\sigma}) \mathbf{I}+\mathbf{s}$, we write the scalar invariants as

$$
p=\frac{1}{3} \operatorname{tr} \boldsymbol{\sigma}, \quad q=\sqrt{\frac{3}{2} \operatorname{tr} \mathbf{s}^{2}}, \quad \zeta=\cos 3 \theta=\frac{\sqrt{6} \operatorname{tr} \mathbf{s}^{3}}{\sqrt{\left(t r \mathbf{s}^{2}\right)^{3}}},
$$

where the invariant $p$ is equal to the mean effective stress or the octahedral normal hydrostatic stress, $q$ is the equivalent shear stress, $\operatorname{tr} \sigma$ is the first principal invariant of the stress tensor, $\frac{1}{2} \operatorname{tr} \mathbf{s}^{2}, \frac{1}{3} \operatorname{tr} \mathbf{s}^{3}$ are the second and third principal invariants of the stress deviator, $\theta$ is the Lode angle, $\zeta$ is the Lode parameter in the stress space. The equivalent shear stress reduces to $q=\left|\sigma_{1}-\sigma_{3}\right|$ for triaxial stress states with $\sigma_{2}=\sigma_{3}$, see [2].

\section{The loading function}

The shape of the loading function surface is illustrated in Fig.1, where its cross-sectional trace in the deviatoric plane and its meridians in the meridian planes are shown. The function is continuous, smooth and convex. Since the surface is closed at both ends, it entirely accounts for effect of the hydrostatic pressure. The apexes of the surface on the hydrostatic axis are assumed to pass through the point $p_{c}$ for the compressive hydrostatic stress and the point $p_{s}$ for the tensile uniform triaxial stress. Using the stress invariants, the loading function is expressed mathematically by the following simple analytical expression

$$
f(p, q, \zeta)=q-A_{o}(\zeta)\left(p_{s}+p\right) \sqrt{p_{c}-p}
$$

where $A_{\delta}(\zeta)$ is a function of the Lode parameter. For the point $(p, q, \zeta)$ that corresponds to the current load level $f(p, q, \zeta)=0$ and $q=A_{o}(\zeta)\left(p_{s}+p\right) \sqrt{p_{c}-p}$.

The traces of the standard Drucker-Prager cones on the meridian planes are also shown in Fig. 1 as the straight lines in the $p-q$ plane, which can be described algebraically by linear equations with the two material constants. Using the most common approximations obtained by making the failure surfaces of the Drucker-Prager and Mohr-Coulomb criteria coincident either at the outer or inner edges of the Mohr-Coulomb surface, these constants can be matched to the cohesion $c$ and the friction angle $\varphi$ of the Mohr-Coulomb failure criterion. Since the ratio $q_{t o} / q_{c o}$ of the two characteristic lengths, the tensile length $q_{t}=q_{t o}$ and compressive length $q_{c}=q_{c o}$, for any deviatoric cross sections of the Mohr-Coulomb

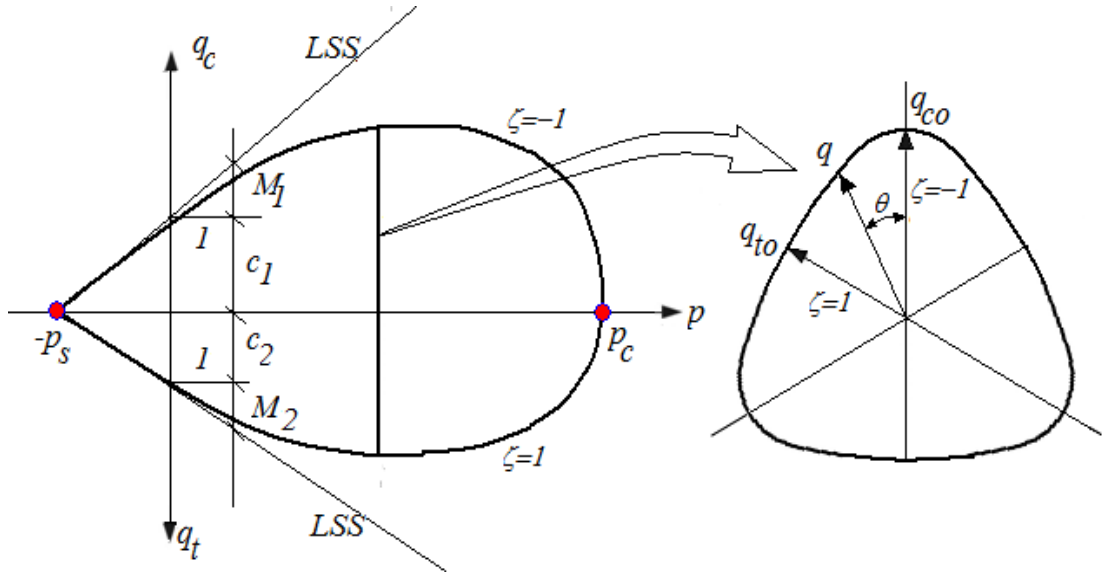

Fig. 1.Cross-sectional shape of the loading surface, LSS - Drucker-Prager limit state surface 
surface is constant, i.e.

$$
g=\frac{q_{t o}}{q_{c o}}=\frac{3-\sin \varphi}{3+\sin \varphi},
$$

the two surfaces of the Drucker-Prager and Mohr-Coulomb failure criteria can be made to agree along the compressive meridian or along the tensile meridian, if they are matched at their apex. For the outer compression cone, which passes through the compressive meridian where $\zeta=-1(\theta=0)$, we have the linear equation $q_{1}=M_{1} p+c_{1}$ with the material constants

$$
M_{1}=2 \sqrt{2} \frac{\sin \varphi}{3-\sin \varphi}, \quad c_{1}=2 \sqrt{2} \frac{\cos \varphi}{3-\sin \varphi} c .
$$

On the other hand, for the inner extension cone, which passes through the tensile meridian where $\zeta=1(\theta=\pi / 3)$, we have the linear equation $q_{2}=M_{2} p+c_{2}$ with two sets of material constants $\left(M_{2}, c_{2}\right)$ and $(c, \varphi)$ related by

$$
M_{2}=2 \sqrt{2} \frac{\sin \varphi}{3+\sin \varphi}, \quad c_{2}=2 \sqrt{2} \frac{\cos \varphi}{3+\sin \varphi} c .
$$

In the loading function (3) we have curved meridians. The trace of the failure surface on deviatoric sections is shown in Fig. 1. It is neither circular nor irregular hexagon and depends on the angle $\theta$ (or the Lode parameter $\zeta$ ). The cross-sectional shape of the loading surface on the deviatoric plane is a curvilinear triangle and it has the standard threefold symmetry with the maximal radius $q_{c}$ on the compressive meridian $(\zeta=-1)$ and the minimal radius $q_{t}$ on the tensile meridian with $(\zeta=1)$. Between these two limiting cases, for $-1<\zeta<1$, the following simple approximation from [3] is used

$$
\rho(\zeta)=\frac{2 g}{1+g+(1-g) \zeta}
$$

and the function $A_{o}(\zeta)$ in (3) can be written as

$$
A_{o}(\zeta)=\frac{\rho(\zeta) M_{1}}{\sqrt{p_{c}+p_{s}}}
$$

The loading function (3) can be matched to the Drucker-Prager failure criterion which can serve as the limit state surface. Matching the coordinates of the apexes we will obtain $p_{s} \approx c_{1} / M_{1}$. From the condition $\partial q / \partial p=\rho(\zeta) M_{1}$ for $p=-p_{s}$ it is possible to obtain the coordinate of the point $p_{c}$ as

$$
p_{c}=\frac{a^{2} p_{s}+p}{1-a^{2}}, \quad a=\frac{q}{\rho(\zeta) M_{1}\left(p+p_{s}\right)} .
$$

It should be noted that the point $(p, q, \zeta)$ of the current loading corresponds to the softening state of the material if it lies outside the limit surface of the Drucker-Prager criterion. 


\section{Hardening and softening behaviour}

The hardening/softening modulus $H$ in the constitutive equation (1) can be calculated in the coordinate system $(p, q, \zeta)$ by means of the matrices $\left[\mathbf{p}_{\sigma}\right]=[p, q, \zeta]^{T}$ and [f] , see [1]

$$
H=-\frac{\partial f}{\partial \kappa}\left[\mathbf{p}_{\sigma}\right]^{T}[\mathbf{f}]
$$

where the scalar $\kappa$ is introduced as the internal measure for hardening/softening of the plastic flow. In the case of work hardening/softening, the scalar $\dot{\kappa}$ is the plastic work measure $\dot{\kappa}=\boldsymbol{\sigma} \cdot \dot{\boldsymbol{\varepsilon}}^{p l}$. Substituting $\kappa$ in the expression (10), we will obtain

$$
H=-[\mathbf{f}]^{T}\left[\overline{\mathbf{D}}_{p}\right]^{T}[\mathbf{f}] \text { and }\left[\overline{\mathbf{D}}_{p}\right]=\left[\begin{array}{ccc}
3 K_{h} & 0 & 0 \\
0 & G_{s} & 0 \\
0 & 0 & 1
\end{array}\right] \text {, }
$$

where $3 K_{h}=\partial p / \partial \varepsilon^{p l}$ and $G_{s}=\partial q / \partial y^{p l}$ are plastic octahedral compressive and shear modules, respectively. We assume that the soil hardening is characterized by the modulus $K_{h}$ whereas the soil softening by the modulus $G_{s}$.

We also assume that the void ratio $e$ is a basic physical characteristic of soil behaviour and can be taken as a hardening internal variable. This variable is changing during the triaxial loading and is indirectly dependent on the octahedral normal stress $p$. The strength of soils increase as the void ratio decreases. Based on the experimental consolidation line shown in Fig. 2 in the $e-\ln p$ plane (the virgin compression line), we have the equation $e=e^{0}-\mu \ln p$ during the active loading and the equation $e=e^{A}-\delta \ln p$ during unloading, where $e^{0}$ is the initial void ratio, $e^{A}$ is the void ratio at the time of unloading, $\mu$ and $\delta$ are the experimental parameters, see $[4,5]$. The volumetric strain $\varepsilon_{V}=3 \varepsilon$ caused by the change of

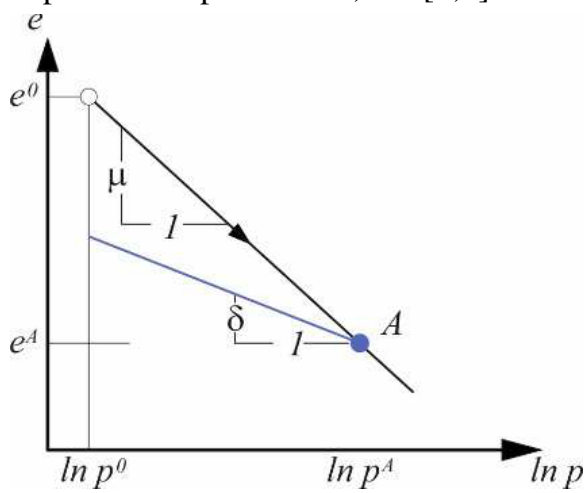

Fig. 2. The change of the void ratio during hydrostatic loading/unloading the void ratio can be written in the form $\varepsilon_{V}=-\left(e-e_{0}\right) /(1+e)$. Considering the standard decomposition $\dot{\varepsilon}^{p l}=\dot{\varepsilon}-\dot{\varepsilon}^{e l}$, where $\dot{\varepsilon}^{e l}$ is the elastic strain, we can write the volumetric plastic strain as

$$
\dot{\varepsilon}_{V}^{p l}=\frac{\mu-\delta}{1+e} \dot{p} .
$$

Based on the relationship (12) the derivative in the hardening modulus (9) can be written as

$$
\frac{\partial f}{\partial \kappa}=-\frac{3(1+e)}{\mu-\delta} \frac{\partial f}{\partial p} \text { and } K_{h}=\frac{1+e}{\mu-\delta}
$$

If the stress state lies outside of the limit state surface (Fig. 1), it cannot be accepted as a solution because of violation of the classical Drucker's postulate about the positive dissipation of energy during the plastic deformation. This is a range of damaged behaviour of the soil that is caused by octahedral shear strains when octahedral shear stresses exceed limit values. However, we can observe in practice that the process of soil deformations continues as a softening of the material. In the $(\gamma-q)$-plane (Fig. $3 b$ ), the softening of the 
soil behaviour is represented by a descending branch of a curve. The derivative of $f$ in the expression (13) can be thus rearrange into a function of new variables as

$$
\frac{\partial f}{\partial \kappa}=\frac{1}{q} \frac{\partial f}{\partial q} \frac{\partial q}{\partial \gamma}=\frac{G_{s}}{q} \frac{\partial f}{\partial q}=\frac{1}{q} \frac{G_{o} G_{t}}{G_{o}-G_{t}} \frac{\partial f}{\partial q},
$$

where $G_{t}$ is the tangent modulus of the octahedral shear strain and $G_{s}$ is the tangent plastic modulus. To determine the modulus $G_{t}$, the curve in the $(\gamma-q)$-plane can be again used, for example with the following analytical expression for the descending and ascending branch

$$
\xi=\frac{q}{\hat{q}}=\frac{\kappa \eta}{1+(\kappa-2) \eta+\eta^{2}},
$$

where $\eta=\gamma / \hat{\gamma}, \hat{\gamma}=\kappa \hat{q} /\left(2 G_{o}\right)$ and peak values of the curve are denoted by $\hat{\gamma}$ and $\hat{q}$.

As shown in Fig. 3a, if the current stress state with coordinates $(p, q)$ lies outside of the limit surface (the point $l$ in Fig. 3a), the softening behaviour, associated with the descending branch of the curve in the $(\gamma-q)$-plane, should be determined by the strain state of soil, e.g. by the octahedral shear strain. The corresponding stress state, as defined by the curve, would be below the limit stress state. This formally means a return to the inside of the surface of the Drucker-Prager yield criterion (the point 2 in Fig. 3a).

(a)
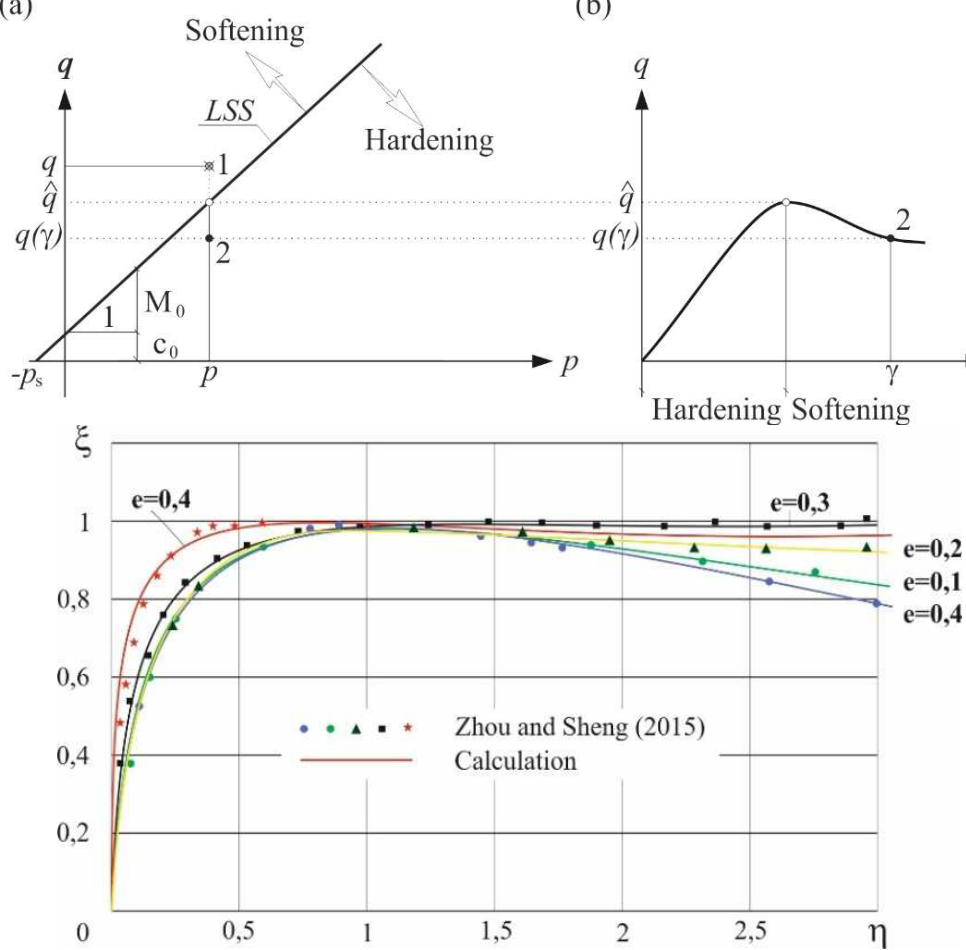

(b)

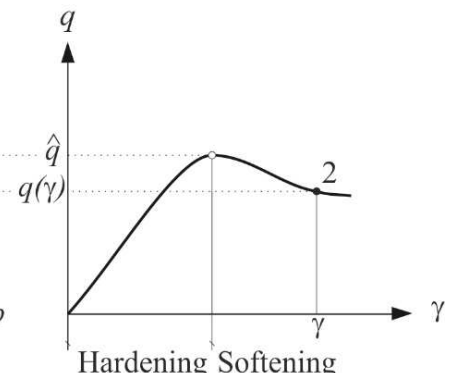

Hardening Softening $\mathrm{e}=\mathbf{0 , 1}$ 0,4 
The parameter $\kappa$ in the equation (15) characterizes the secant modulus of the shear strain. It depends on the void ratio of the soil. Based on an analysis of experimental data $[6,7]$ the following expression can be recommended

$$
\kappa=\kappa_{p}-\left(\kappa_{p}-1\right) \exp (-0.1 e)
$$

where $\kappa_{p}$ is an experimental parameter $\left(\kappa_{p}=2 \div 6\right)$. Figure $3 \mathrm{c}$ shows curves drawn per the expression (15) with (16) for different values of $e$ and with a comparison to the experimental data from [7].

Taking the derivative of the function (15) with respect to $\gamma$, we will receive

$$
G_{t}=\Omega G_{0}
$$

where the nonlinearity function $\Omega$ equals to

$$
\Omega=\frac{1-\eta^{2}}{\left[1+(\kappa-2) \eta+\eta^{2}\right]^{2}} .
$$

\section{Dilatancy}

The phenomenon of dilatancy during plastic flow is observed for many geomaterials. The associative plasticity usually employs as flow potential the classical yield function of the standard Drucker-Prager cone. However, the description of dilatancy in the framework of the theory of the plastic flow based on the relationship (1) is impossible because of violation of the classical Drucker's postulate about the positive dissipation of energy during the plastic deformation. To derive the appropriate flow rule one can write the plastic flow law by means of two equations of stress invariants, the one for the volumetric plastic strain and the second one for the octahedral shear plastic strain in the form [8]

$$
\dot{\varepsilon}^{p}=\lambda_{1} \frac{\partial f}{\partial p}, \quad \dot{\gamma}^{p}=\lambda_{2} \frac{\partial f}{\partial q} .
$$

Hence, considering the expression $\left(13_{2}\right)$, we can write the following relationship

$$
k_{d}=\frac{\dot{\varepsilon}_{p}}{\dot{\gamma}_{p}}=\frac{\lambda_{1} \partial f / \partial p}{\lambda_{2} \partial f / \partial q}=\lambda_{o} \frac{\partial f}{\partial p}=\lambda_{o} \frac{p_{s}-2 p_{c}+3 p}{2\left(p_{s}+p\right)\left(p_{c}-p\right)} q,
$$

where for the materials without dilatancy we have $\lambda_{0}=0$ and for the dilatant materials we have $\lambda_{0}>0$. The expression of the parameter $\lambda_{o}$ as a function of the initial void ratio $e$ can be established by means of the experimentally determined results from [2] and [7].The following expression can be recommended

$$
\lambda_{o}=\frac{0.0025}{1-0.95 e}
$$

\section{Validation}

The use of the model for the soil mechanics problems is validated next by a comparison with results available in the literature. In the first example, drained triaxial tests from [2] are investigated. The results of experimental data from [7] are compared with calculations of 
the own model for three types of soils with different density state: a loose state, a medium state and a dense state. The material parameters of the model used in the calculations are given in Table 1. The analyses are carried out with loading control and a step size of the value $\Delta \sigma_{z}=0.02[\mathrm{MPa}]$ until reaching the critical state of the soil. In the first step, the hydrostatic stress state is introduced with the value of $\sigma_{h}=0.1[\mathrm{MPa}]$.The comparison between numerical and experimental results is given in Fig. 4 in diagrams of the difference of principal stresses versus vertical axial strain (Fig. 4a) and the volumetric strain-vertical axial strain diagrams (Fig. 4b). Good agreement is found. The response of the model in the triaxial compression test is quite realistic.

Table 1. Material parameters for triaxial tests

\begin{tabular}{|c|c|c|c|c|c|c|c|}
\hline $\begin{array}{c}\text { Density } \\
\text { state }\end{array}$ & $E[M P a]$ & $v[-]$ & $\varphi\left[{ }^{0}\right]$ & $c[M P a]$ & $\mu[-]$ & $\delta[-]$ & $e[-]$ \\
\hline Dense & 40 & 0.2 & 40 & 0 & 0.09 & 0.05 & 0.3 \\
\hline Medium & 30 & 0.2 & 35 & 0 & 0.09 & 0.05 & 0.8 \\
\hline Loose & 20 & 0.2 & 30 & 0 & 0.09 & 0.05 & 0.9 \\
\hline
\end{tabular}

(a) $\mathrm{q}[\mathrm{MPa}]$
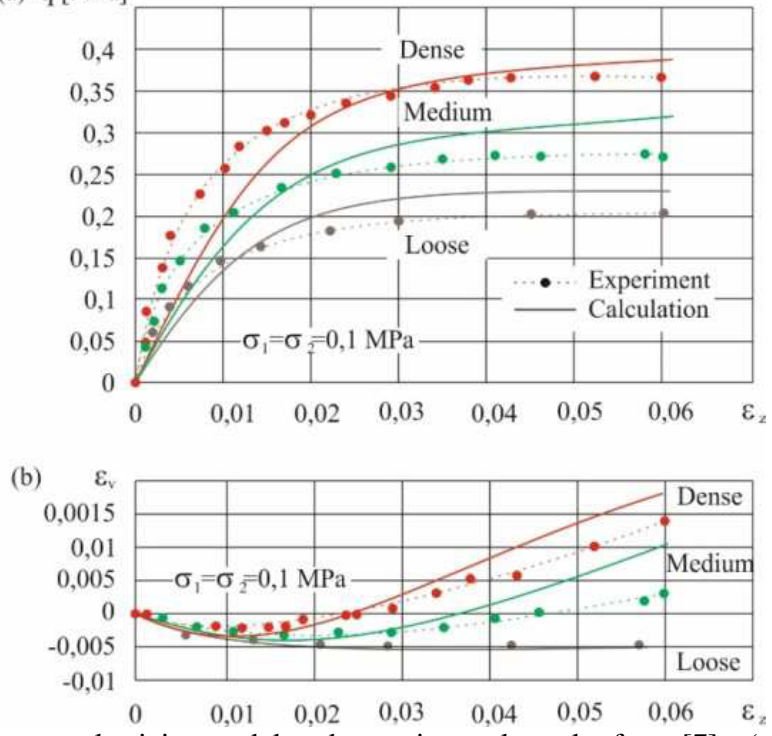

Fig.4. Comparison between plasticity model and experimental results from [7]: (a) equivalent shear stress versus vertical strain; (b) volumetric strain versus vertical strain.

In the second examples, deformations of the porous soil under the triaxial compression are investigated for various values of the initial hydrostatic stress. The comparison with the experimental results from [9] is shown in Fig. 5 in the similar diagrams as in the first example. The following material parameters are taken: $E=9.75[\mathrm{MPa}], v=0.25$, $\varphi=34^{\circ}, c=0[M P a], \mu=0.1, \delta=0, e=0.61$. The numerical analyses are conducted for the four values of the initial hydrostatic stress: $\sigma_{h}=0.1 ; 0.3 ; 1.05 ; 2.0[\mathrm{MPa}]$ in the first step of the loading. The calculations are carried out with loading control and a step size of the value $\Delta \sigma_{z}=0.02[\mathrm{MPa}]$ in the subsequent steps until reaching the critical state of the soil. Good agreement is again found. The response in the triaxial compression test confirmed utility of the model. 
(a) $\mathrm{q}[\mathrm{MPa}]$

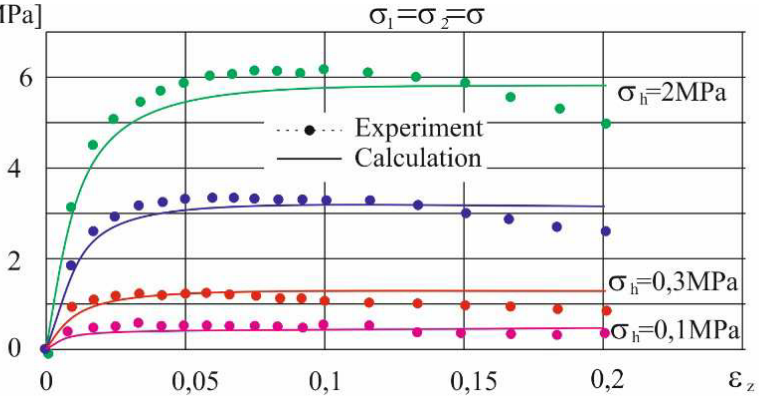

(b)

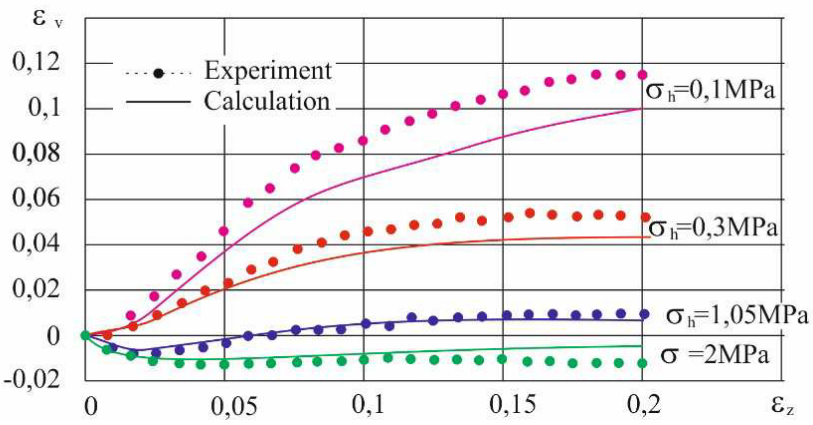

Fig.5. Comparison between plasticity model and experimental results from [9]: (a) equivalent shear stress versus vertical strain; (b) volumetric strain versus vertical strain.

\section{References}

1. O. Zienkiewicz, A.H.C. Chan, M. Pastor, T. Shiomi, Computational Geomechanics with special Reference to Earthquake Engineering (London: John Wiley \& Sons, 1999)

2. R.B.J. Brinkgreve, P.A. Vermeer, Plaxis. Finite Element Code for Soil and Rock Analysis. Materials Models Manual. Part 3 (Rotterdam: Balkema, Brookfild, 1998)

3. J.H. Argiris, G. Faust, J. Szimmat, P. Warnke, K. Willam, Nuclear Engineering and Design, 28, 42 (1974)

4. K. J. Roscoe, A. N. Schofield, Proc. European Conf. on Soil Mechanics and Foundation Engineering, 1, 47 (1963)

5. K. Terzaghi, R.B. Peck. Soil Mechanics in Engineering Practice. 2nd ed. (New York: John Wiley \&Sons, 1967)

6. D. Masin, Int. J. Num. Anal. Meth. Geomech., 34, 73 (2010)

7. A. Zhou, D. Sheng, Comput. Geotech., 63, 46 (2015)

8. R. de Borst, A. E. Groen. 2 Computational Strategies for Standard Soil Plasticity Models. Modeling in Geomechanics (Edited by Zaman M, Booker J, Gioda G., John Wiley \& Sons, 2000).

9. K.L. Lee, H.B. Seed, J. Soil. Mech. Found. Div., ASCE, 93(SM6), 117 (1967) 